\title{
ABELIAN SUBGROUPS OF TOPOLOGICAL GROUPS ${ }^{1}$
}

\author{
BY
}

\author{
SIEGFRIED K. GROSSER AND WOLFGANG N. HERFORT
}

\begin{abstract}
In [1] Šmidt's conjecture on the existence of an infinite abelian subgroup in any infinite group is settled by counterexample. The well-known Hall-Kulatilaka Theorem asserts the existence of an infinite abelian subgroup in any infinite locally finite group. This paper discusses a topological analogue of the problem. The simultaneous consideration of a stronger condition-that centralizers of nontrivial elements be compact - turns out to be useful and, in essence, inevitable. Thus two compactness conditions that give rise to a profinite arithmetization of topological groups are added to the classical list (see, e.g., [13 or 4]).
\end{abstract}

1. Introduction. The well-known example by Adian and Novikov [1] invalidates both the Burnside conjecture and a conjecture by O. Yu. Šmidt: "There is an infinite abelian subgroup in every infinite group." Thus the Šmidt conjecture becomes a restrictive condition. One is far from knowing all groups that satisfy it; it is, however, satisfied (e.g.) for locally finite groups (theorem of Hall and Kulatilaka [8]). For compact groups, a negative answer to the question posed above would imply a negative answer to the restricted Burnside problem (see [10]). A stronger condition - that nontrivial elements possess finite centralizers-reduces the investigation to one for pro-p-groups (see (1.1)).

It is the purpose of this paper to study topological analogues of Šmidt's question. In general, the method of topologizing problems of the discrete theory provides perspective and allows for the development of a more comprehensive theory in which techniques of Lie theory and discrete theory can profitably be combined. Thus the topological versions of finiteness conditions (see especially [16, Chapter 4]) have given rise to the rather extensive theory of compactness conditions (see [13]).

After some preliminary lemmas in $\$ 2$ (which are needed in $\$ \S 3,4$ ) we study Lie groups in which all abelian subgroups have compact closure. It turns out that these Lie groups are extensions of connected compact groups by discrete groups satisfying the above condition (see (3.2)). The main results are contained in §4. Moore groups whose nontrivial elements have compact centralizers are either compact or are finite extensions of Moore $p$-groups (see (4.3)). The proof of (4.3) is of a rather technical nature. A by-product of the investigation is the result that a group $G$ possessing the

\footnotetext{
Received by the editors April 4, 1983.

1980 Mathematics Subject Classification. Primary 22A05, 22D05.

Key words and phrases. Compactness conditions, profinite theory, Lie groups, Moore groups.

${ }^{1}$ The results of this paper were announced in Math. Rep. Acad. Sci. Canada 4 (1982), 249-254 and presented at the Conference on Group Theory held at Oberwolfach, Federal Republic of Germany, May $2-5,1983$.
} 
above centralizer condition and for which $G_{0} \in[\mathrm{SIN}]_{G}$ is itself compact or totally disconnected (see (4.2)). Two pertinent examples given in $\$ 5$ delimit the scope of the results obtained.

We next give the definitions of the classes of locally compact groups needed in the paper. $\mathfrak{B}$ denotes a subgroup of the (topological) automorphism group Aut $G$ :

[IN] :=class of locally compact groups $G$ possessing a compact $G$-invariant neighborhood of $e$.

$[\mathrm{SIN}]_{\mathcal{H}}:=$ class of locally compact groups $G$ possessing a fundamental system of $\checkmark B$-invariant neighborhoods of $e$.

$[\mathrm{FC}]_{\text {H }}^{-}:=$class of locally compact groups $G$ with precompact $\mathfrak{B}$-orbits.

[M] $:=[$ Moore] $:=$ class of locally compact groups all of whose irreducible Hilbert space representations are finite dimensional.

[Z] :=class of locally compact groups $G$ with $G / Z(G)$ compact.

[Lie] :=class of Lie-groups.

Aside from these standard classes (see [4 and 13]) we employ the following notation.

$[\mathrm{P}]^{-} \quad:=$ class of locally compact periodic groups.

[TD] :=class of locally compact totally disconnected groups.

$\Omega \quad:=[\mathrm{IN}] \cap[\mathrm{P}]^{-} \cap[\mathrm{TD}]$. (Because of [11, Lemma 4.2, p. 408] one has $[\mathrm{M}] \cap$ $[\mathrm{P}]^{-} \cap[\mathrm{TD}] \subseteq \Omega$.)

$[\mathrm{LF}]^{-}:=$class of locally compact topologically locally finite groups $(G \in$ $[\mathrm{LF}]^{-}: \Leftrightarrow$ each precompact subset of $G$ generates a precompact subgroup).

[K] :=class of compact groups.

Furthermore, we now formalize the two finiteness conditions and the corresponding compactness conditions, as follows.

[AF] :=class of abstract groups $G$ whose abelian subgroups are finite.

[CF] :=class of abstract groups $G$ with finite centralizers $C_{G}(x)$ of the elements $x \neq e$.

$[\mathrm{AF}]^{-} \quad:=$ class of locally compact groups $G$ whose closed abelian subgroups are compact.

$[\mathrm{CF}]^{-}:=$class of locally compact groups $G$ with compact centralizers $C_{G}(x)$ of the elements $x \neq e$.

One has $[\mathrm{CF}] \subset[\mathrm{AF}]$ and $[\mathrm{CF}]^{-} \subset[\mathrm{AF}]^{-}$(see (5.1)). The two questions referred to above, whether or not $[\mathrm{CF}] \cap[\mathrm{K}]=[\mathrm{AF}] \cap[\mathrm{K}]=$ class of finite groups, are open [10]. The question of whether or not an infinite pro-p-group in [CF] actually exists is also still open.

By $N_{G}(H)$ we denote the normalizer of $H \subseteq G$, by $H^{x}$ and $H^{\beta}$, respectively, for $x \in G$, and $\beta \in$ Aut $G$, the image $x H x^{-1}$ and $\beta(H)$, respectively; by $\exp G$ the exponent of $G$. For a prime $p$ and an infinite index set $I$, let $\left(C_{p}^{I}\right)^{*}$ denote the weak direct product of $|I|$ copies of the cyclic group $C_{p}$. By a "sequence" we mean a short 
exact sequence of topological groups, $K>G \stackrel{\pi}{\rightarrow} H$, where $H=\pi(G) \cong G / K$. For $\mathfrak{B} \leqslant$ Aut $G$ let $C_{G}(\mathfrak{B}):=\{g \in G \mid \beta(g)=g$ for all $\beta \in \mathfrak{B}\}, E:=\{e\}$.

In order to elucidate the techniques used in the paper we close $\S 1$ with a result on groups in $[\mathrm{CF}] \cap[\mathrm{K}]$.

(1.1) Proposition. Let $G \in[\mathrm{CF}] \cap[\mathrm{K}]$. Then $G$ is either finite or a pro-p-group for an odd prime $p$ (see [15] for the definition of a pro-p-group).

Proof. Clearly $G \in[\mathrm{CF}]$ implies $G$ is a torsion group; hence its identity component, $G_{0}$, a projective limit of connected compact Lie torsion groups, is trivial, so $G$ is profinite. If $G$ is not a pro-p-group there exist an open $N \unlhd G$ and two primes $p \neq q$ such that $p$ and $q$ divide $|G / N|$. If all Sylow subgroups are finite, it follows from this, on account of [10, Theorem 2, p. 460], that $G$ is finite.

Let $r$ be a prime such that $N$ contains an infinite $r$-Sylow subgroup $R$. It follows from the Frattini argument (see e.g. (2.4)(1)) that there exists a prime $s \neq r$, $s \in\{p, q\}$, and an element $x \in N_{G}(R)$ of order $s$ such that $x^{-1} R x=R$. Let

$$
L:=\langle x, R\rangle \text { and } \Phi:=\left\{M=M^{-} \leqslant R \mid[R: M]<\boldsymbol{\aleph}_{0} \text { and } x^{-1} M x=M\right\} .
$$

$\Phi$ constitutes a base of $e$-neighborhoods in $R$. If $x$ acts fixed-point-free on $M, M$ is nilpotent [6, Theorem 2, p. 405] and, being a torsion group, is locally finite; but $M \notin[\mathrm{AF}]$, because of $[\mathbf{8},(2.5)$, p. 72], a contradiction. Hence there exists, in every $M \in \Phi$, a $y_{M}$ with $\left[x, y_{M}\right]=e$, i.e., $C_{G}(x)$ is infinite, a contradiction. That $p$ cannot be 2 follows from $[8,(2.5)$, p. 72].

As this proof suggests, one may, in the situations under consideration in this paper, successfully employ techniques of the theory of (pro-)finite groups-coprimeness conditions and Sylow theory [15], i.e., a certain arithmetization [14]. Other tools are generalizations of the above Frattini argument as well as theorems on the lifting of fixed points of automorphism groups [6] and special methods of approximation by Lie groups [5].

\section{Auxiliary lemmas.}

Definition. For $G \in \Omega$ call $x \in G$ a $p$-element $\Leftrightarrow\langle x\rangle^{-}$is a pro-p-group (see [14]). Let $\Pi(G):=\{p \mid \exists$ a $p$-element $x \in G \backslash\{e\}\}$.

(2.1) Lemma. Let $\left\{x_{\nu}\right\}$ be a net of p-elements in $G$, and $x_{0}=\lim _{\nu} x_{\nu}$. Then $x_{0}$ is a p-element.

Proof. $G \in \Omega \Rightarrow K>G \rightarrow D$, where $K$ is compact and open, and $D$ is a discrete torsion group [4]. Since $\left\langle x_{0}, K\right\rangle=\left\langle x_{0}\right\rangle K$ is an open $x_{0}$-neighborhood $x_{\nu} \in\left\langle x_{0}, K\right\rangle$ for $\nu \geqslant \nu_{0}$. It therefore suffices to assume that $G$ is compact, i.e., profinite. Now use [6, Lemma 6].

In what follows we shall also need certain facts concerning the topological analogue of the class of locally finite groups.

REMARK. $G \in[\mathrm{LF}]^{-} \Leftrightarrow$ every precompact $e$-neighborhood generates a compact subgroup. It is easy to show that for $G / G_{0}$ discrete one has $G \in[\mathrm{LF}]^{-} \Leftrightarrow G / G_{0}$ is locally finite and $G_{0}$ is compact. 
(2.2) Lemma. (1) $G \in[\mathrm{LF}]^{-}$and $H=H^{-} \leqslant G \Rightarrow H \in[\mathrm{LF}]^{-}$.

(2) $N \gg G \rightarrow L$ with $N, L \in[\mathrm{LF}]^{-} \Rightarrow G \in[\mathrm{LF}]^{-}$.

Proof. (2) Let $X$ be a compact symmetric $e$-neighborhood in $G$. It suffices to show that $\langle X\rangle$ is compact. We have $\langle X\rangle N / N=\langle X N / N\rangle$, and the latter group is compact because $X N / N$ is a compact $e$-neighborhood of $L \in[\mathrm{LF}]^{-}$. For $r \in \mathbf{N}$ the collection $\left\{(X N)^{r} / N\right\}=\left\{X^{r} N / N\right\}$ is an open covering of $\langle X\rangle N / N$, so there exists an $r_{0}$ with $X^{r_{0}} N / N=\langle X\rangle N / N$. Therefore $X^{r_{0}}$ contains a system of representatives of $\langle X\rangle N / N$, hence also one of the isomorphic group $\langle X\rangle /\langle X\rangle \cap N$. This implies

$$
X^{r_{0}}(\langle X\rangle \cap N)=\langle X\rangle \supseteq X^{2 r_{0}} .
$$

Let $S:=X^{3 r_{0}} \cap N \leqslant N$. From the relation $X^{2 r_{0}} \subseteq X^{r_{0}} S \subseteq X^{r_{0}}\langle S\rangle$, one obtains, by induction on $k, X^{k r_{0}} \subseteq X^{r_{0}}\langle S\rangle$ and, from this, $\langle X\rangle \subseteq X^{r_{0}}\langle S\rangle^{-} . N \in[\mathrm{LF}]^{-}$now implies $\langle S\rangle^{-}$is compact.

(2.3) Corollary. $G \in[\mathrm{M}] \cap[\mathrm{P}]^{-} \Rightarrow G \in[\mathrm{LF}]^{-}$.

Proof. There exists a sequence $K \gg G \rightarrow H$, where $K$ is compact and $H \in[\mathrm{M}]$ $\cap[\mathrm{Lie}] \cap[\mathrm{P}]^{-}$; according to [11, Theorem 3] there exists a sequence $M>H \rightarrow F$, where $F$ is finite and $M \in[\mathrm{Z}]$, so $M \in[\mathrm{LF}]^{-}$also.

(2.4) Lemma. (1) (Frattini Argument). Let $K$ be a profinite closed normal subgroup of $G$ and $P$ a p-Sylow subgroup of $K$. Then $G=N_{G}(P) K$.

(2) Assume $G$ is locally compact, $G_{0}$ compact, $T$ a maximal torus (i.e. a maximal connected abelian closed subgroup) of $G_{0}$. Then $G=N_{G}(T) G_{0}$.

Proof. (1) Let $g \in G$. If $P$ is a $p$-Sylow subgroup of $K$, then so is $P^{g}$. On account of the conjugacy of such subgroups [17] there exists $k \in K$ with $P^{g}=P^{k}$. From $g k^{-1} \in N_{G}(P)$, the assertion follows.

(2) It follows from [9, Lemma 1.3, p. 5] and from properties of projective limits that there exist maximal tori in connected compact groups and that any two are conjugate. Now proceed as in (1).

(2.5) LemmA. Assume $K \gg G \rightarrow D, K$ compact and open, $D$ discrete. If $G \in[\mathrm{AF}]^{-}$, then $D$ is a torsion group which does not contain a locally cyclic subgroup. If, in addition, $D$ is abelian, then $|\Pi(D)|$ is finite; furthermore, if $D$ is infinite, then there exists a prime $p$ such that $D$ possesses an infinite subgroup of the form $\left(C_{p}^{I}\right)^{*}$, where I is an infinite index set.

ProOF. If $D$ is not a torsion group then there exists $K>G_{1} \stackrel{\pi}{\rightarrow} \mathbf{Z}$ with $G_{1}=G_{1}^{-} \leqslant$ $G$, which means $G_{1} \in[\mathrm{AF}]^{-}$. Let $t \in G_{1}, \pi(t)=1 \in \mathbf{Z}$. Then $\langle t\rangle^{-}$would not be compact, a contradiction. Assume $D$ contains a locally cyclic (hence infinite) subgroup $L=\cup_{n \in \mathbf{N}} L_{n}, L_{n} \subset L_{n+1}$, each $L_{n}:=\left\langle\dot{x}_{n}\right\rangle$ finite and cyclic and $n \in \mathbf{N}$. Then there exists $K \gg H \rightarrow L$ with $H \in[\mathrm{AF}]^{-}$, and there are numbers $\alpha_{n r}$ with $\dot{x}_{n}^{\alpha_{n r}}=\dot{x}_{r}($ for $n>r)$ and $\alpha_{n r} \alpha_{r s}=\alpha_{n s}\left(\right.$ for $n>r>s$ ). For each $n$ let $x_{n} \in \dot{x}_{n}$ be a 
representative. We now construct inductively monothetic subgroups $Z_{n}:=\left\langle z_{n}\right\rangle^{-}$of $H$ with the following properties.

(1) $\dot{z}_{n}=\dot{x}_{n}$ and

(2) $Z_{n} \supset Z_{n-1}$.

Let $Z_{0}:=E$ and let $\{t\}$ be a strictly increasing sequence of natural numbers such that the sequence $\left\{x_{t}^{\alpha_{t 1}}\right\}_{t}$, which lies in the compact set $\dot{x}_{1}$, converges to a limit $z_{1}$ (so $\left.\dot{z}_{1}=\dot{x}_{1}\right)$. Put $Z_{1}:=\left\langle z_{1}\right\rangle^{-}$. Assume we have already found $z_{1}, z_{2}, \ldots, z_{n}$ and $Z_{1}, Z_{2}, \ldots, Z_{n}$ with properties (1) and (2) such that $\lim _{t} x_{t}^{\alpha_{t j}}=z_{j}(1 \leqslant j \leqslant n)$.

Replacing $\{t\}$, if necessary, by a subsequence, one may assume $\left\{x_{t}^{\alpha_{t, n+1}}\right\}_{t}$ to be convergent in $\dot{x}_{n+1}$, with limit $z_{n+1} \in \dot{x}_{n+1}$. We have

$$
\left(x_{t}^{\alpha_{t, n+1}}\right)^{\alpha_{n+1, n}}=x_{1}^{\alpha_{t, n}} \text {. }
$$

Hence $t \rightarrow \infty$ results in $z_{n+1}^{\alpha_{n+1, n}}=z_{n}$, i.e. $\left.Z_{n+1}:=\left\langle z_{n+1}\right\rangle^{-}\right\rangle Z_{n}$. Let $Z:=$ $\left(\cup_{n \in N} Z_{n}\right)^{-}$. Since $\pi(Z)=L$, the closed abelian subgroup $Z \leqslant H$ cannot be compact, a contradiction. Next let $D$ be abelian and $|\Pi(D)|=\aleph_{0}$. We have $D \cong \Pi^{*} D_{p}$, where $p \in \Pi(D)$ and $D_{p}$ denotes the $p$-component of $D$. Choose $x_{p} \in D_{p} \backslash E$ such that $x_{p}^{p}=e$ and put

$$
L_{n}:=\left\langle\prod x_{p} \mid p<n, p \in \Pi(D)\right\rangle .
$$

Then $L:=\cup_{n \in \mathbf{N}} L_{n}$ is locally cyclic in $D$, a contradiction. Therefore $\Pi(D)$ must be finite. Now, if $|D| \geqslant \aleph_{0}$ and $D \cong \prod_{p \in \operatorname{II}(D)}^{*} D_{p}=\prod_{p \in \operatorname{II}(D)} D_{p}$, then there exists $p \in$ $\Pi(D)$ with $\left|D_{p}\right| \geqslant \boldsymbol{\aleph}_{0}$. According to the above, $D_{p}$ contains no quasicyclic subgroup $\mathbf{Z}\left(p^{\infty}\right)$, since the latter is locally cyclic. Thus $D_{p}$ contains no divisible abelian subgroup, hence is reduced. Because of $[2,77.5$, p. 65] there exist an infinite set $I$ and nontrivial subgroups $\left(A_{i}\right)_{i \in I}$ in $D_{p}$ as well as a subgroup $A \leqslant D_{p} A \cong \prod_{i \in I}^{*} A_{i}$. In $A_{i} \backslash E$ we select $a_{i}$ with $a_{i}^{p}=e$. Then $\left\langle\left\{a_{i}\right\}_{i \in I}\right\rangle \cong\left(C_{p}^{I}\right)^{*}$ is an infinite subgroup.

3. The class $[\mathrm{AF}]^{-}$. The examples given in [1 and 12] show that there exist rather complicated noncompact groups in $[\mathrm{SIN}] \cap[\mathrm{AF}] \subset[\mathrm{SIN}] \cap[\mathrm{AF}]^{-}$. Since these groups are discrete it is conceivable that nondiscreteness of a group together with the condition $[\mathrm{AF}]^{-}$results in a more serious restriction. Along these lines we have the following result.

(3.1) Proposition. Let $G \in[\mathrm{IN}] \cap[\mathrm{AF}]^{-}$. Then there is a sequence $K>G \rightarrow D$, in which $K$ is compact and open, and $D$ is a discrete torsion group not possessing any locally cyclic subgroup.

Proof. The structure theorem for [IN]-groups [4] yields a sequence $H>G \rightarrow D$, where $H$ is an extension of a compact group $K$ by a vector group $V$. However, $V$ must be trivial since $G$ is periodic, so $H=K$. By the same structure theorem, $K$ is open. The remaining statement follows immediately from (2.5).

For Lie groups a reduction of the problem to the corresponding problem for discrete groups can be accomplished as follows.

(3.2) THEOREM. For a Lie group $G$ the following holds: $G \in[\mathrm{AF}]^{-} \Leftrightarrow G_{0}$ is compact and $G / G_{0} \in[\mathrm{AF}]$. 
For the proof we need an auxiliary result.

(3.3) Lemma. Assume $I$ is an infinite index set, $p$ a prime, $V:=\left(C_{p}^{I}\right)^{*}, r \in \mathbf{N}$, and $f: V \times V \rightarrow C_{p}^{r}$ a bilinear form with respect to $\mathrm{GF}(p)$ satisfying $f(x, x)=0$ for all $x \in V$. Then there exists an infinite subspace $U \leqslant V$ such that $u_{1}, u_{2} \in U \Rightarrow f\left(u_{1}, u_{2}\right)$ $=0$.

Proof. For $x \in V$ the set $f(x, V)$ is finite; hence there exist an infinite subset $S \subseteq V$ and $t \in C_{p}^{r}$ such that $f(x, S)=\{t\}$. Pick $s_{0} \in S$. We have $f\left(x, S-s_{0}\right)=$ $f(x, S)-f\left(x, s_{0}\right)=0$, so there exists an infinite orthogonal $f$-complement $\{x\}^{\perp}$ of $x$ and $x \in\{x\}^{\perp}$. For $x_{1} \in V, x_{1} \neq 0$, put $U_{1}:=\left\langle x_{1}\right\rangle, V_{1}:=U_{1}^{\perp}$. Inductively we construct the subspaces

$$
U_{n}=\left\langle x_{1}, x_{2}, \ldots, x_{n}\right\rangle, \quad \operatorname{dim} U_{n}=n, \quad V_{n}=U_{n}^{\perp}, \quad\left|V_{n}\right| \geqslant \boldsymbol{\aleph}_{0} .
$$

Pick $x_{n+1} \in V_{n}$ with $\operatorname{dim}\left\langle U_{n}, x_{n+1}\right\rangle=n+1$. Put

$$
U_{n+1}:=\left\langle U_{n}, x_{n+1}\right\rangle, \quad V_{n+1}:=U_{n+1}^{\perp} .
$$

According to the above, the orthogonal complement $\left.W:=\left\{x_{n+1}\right\}\right\}_{V_{n}}^{\perp}$ with respect to $f \mid V_{n} \times V_{n}$ is already an infinite subspace of $V_{n}$. But we have $W \leqslant V_{n+1}$, so that $f\left(x_{n+1}, W\right)=0$ and $f\left(U_{n}, W\right)=0$. Therefore $V_{n+1}$ is infinite.

Proof of (3.2). $(\Leftarrow)$ Let $A=A^{-} \leqslant G$ be abelian and noncompact. Then $\pi(A)$, where $\pi: G \rightarrow G / G_{0}$, is obviously an infinite abelian subgroup of $G / G_{0}$, a contradiction.

$(\Rightarrow)$ First we note that $G_{0} \in[\mathrm{AF}]^{-}$, so that $G_{0}$ is compact [7, p. 180]. Let $T$ be a maximal torus in $G_{0}$. It suffices, first, to show that $N_{G}(T) / T \in[\mathrm{AF}]$. For we have $G_{0}=\cup_{g \in G_{0}} T^{g}$ and therefore $G=N_{G}(T) G_{0}((2.4)(2))$. Thus

$$
\begin{aligned}
G / G_{0} & \cong N_{G}(T) G_{0} / G_{0} \cong N_{G}(T) / N_{G}(T) \cap G_{0} \\
& =N_{G}(T) / N_{G_{0}}(T) \cong\left(N_{G}(T) / T\right) /\left(N_{G_{0}}(T) / T\right) .
\end{aligned}
$$

Since $N_{G_{0}}(T) / T=: W$ is the (finite) Weyl group of $G_{0}$, the sequence $W>$ $N_{G}(T) / T \rightarrow G / G_{0}$ represents a discrete extension with finite kernel. If there exists an infinite abelian subgroup $A^{*} \subseteq G / G_{0}$, then there is the sequence $W>$ $A / T \rightarrow A^{*}$ of locally finite groups. Hence $A / T$ is locally finite and, according to [8, (2.5), p. 72], possesses an infinite abelian subgroup $A_{1} / T \leqslant A / T \leqslant N_{G}(T) / T$, a contradiction to $N_{G}(T) / T \in[\mathrm{AF}]$. In order to prove the assertion, we deduce a contradiction from the assumption $N_{G}(T) / T \notin[\mathrm{AF}]$. Thus assume $H / T \leqslant N_{G}(T) / T$ is an infinite abelian subgroup. Since $H \in[\mathrm{AF}]^{-}$, we deduce from (2.5), for $K:=T, G:=H, D:=H / T$, that there exists a prime $p$ and an infinite index set $I$ such that $\left(C_{p}^{I}\right)^{*} \leqslant H / T$. To this corresponds the sequence $T>H_{1} \rightarrow\left(C_{p}^{I}\right)^{*}$. Let $\varphi: H_{1} \rightarrow$ Aut $T$ be the canonical action of $H_{1}$ on $T$. Since $T \leqslant \operatorname{ker} \varphi$ and $\exp \varphi\left(H_{1}\right)$ $\leqslant p, \varphi\left(H_{1}\right)$ is a torsion subgroup of Aut $T \cong \operatorname{Sl}(n, \mathbf{Z})$, where $n=\operatorname{dim} T$. On account of [5, 1.13 Satz, p. 329] one has $\varphi\left(H_{1}\right)$ finite. For $H_{2}:=\operatorname{ker} \varphi$ one therefore has the sequence $T>H_{2} \rightarrow\left(C_{p}^{I}\right)^{*}$, where $T \leqslant Z\left(H_{2}\right)$ and $H_{2}^{\prime} \leqslant T$, so $H_{2}$ is nilpotent of class $\leqslant 2$. Therefore, for $h^{*}, h \in H_{2}$, one has (since $\left.h^{* p} \in T \leqslant Z\left(H_{2}\right)\right) e=\left[h^{p}, h^{*}\right]$ $=\left[h, h^{*}\right]^{p}$, so $\exp H_{2}^{\prime} \leqslant p$. It follows immediately that $H_{2}^{\prime} \cong C_{p}^{r}$ with $r \leqslant n$. Because of $H_{2}=H_{2}^{-} \leqslant H_{1}=H_{1}^{-} \leqslant G$, one has $H_{2} \in[\mathrm{AF}]^{-}$. 
On the vector space $H_{2} / T \times H_{2} / T$ over $\mathrm{GF}(p)$ we define a $C_{p}^{r}$-valued bilinear form by $f(\dot{x}, \dot{y}):=[x, y]$. It is well defined because $T \leqslant Z\left(H_{2}\right)$, and one has $f(\dot{x}, \dot{x})=0 \in C_{p}^{r}$. By (3.3) there exists $U \leqslant\left(C_{p}^{I}\right)^{*}=H_{2} / T$ with $|U| \geqslant \aleph_{0}$ and $f(U, U)=\{0\}$. From this we get the sequences $T>H_{3} \rightarrow U$, where $H_{3}$ represents a noncompact closed subgroup of $G$. For $x, y \in H_{3}$, one has $[x, y]=f(\dot{x}, \dot{y})=0$, so $\mathrm{H}_{3}$ is also abelian, a contradiction to $\mathrm{H}_{3} \in[\mathrm{AF}]^{-}$.

(3.4) Corollary. $G / G_{0} \in[\mathrm{LF}]$ and $G \in[\mathrm{AF}]^{-}$imply $G / G_{0}$ is finite.

Proof. It follows from $G \in[\mathrm{AF}]^{-}$that $G_{0}$ is compact and $G / G_{0} \in[\mathrm{AF}]$. Now, if $\left|G / G_{0}\right| \geqslant \boldsymbol{\aleph}_{0}$, then it follows from $G / G_{0} \in[\mathrm{LF}]$ and from $[8,2.5$, p. 72$]$ that $G / G_{0}$ possesses an infinite abelian subgroup, a contradiction.

4. The classes $[\mathrm{CF}]^{-} \cap[\mathrm{SIN}]$ and $[\mathrm{CF}]^{-} \cap[\mathrm{M}]$. For the proofs of the structure theorems (4.2) and (4.3) we need some information concerning the lifting of fixed points of groups $\mathfrak{B}$ of automorphisms in certain $\mathfrak{B}$-invariant sequences for $G$.

(4.1) Proposition. For $\mathfrak{B} \leqslant$ Aut $G$, and $G \in[\mathrm{SIN}]_{\mathfrak{B}} \cap[\mathrm{K}]$, let $K>G \stackrel{\pi}{\rightarrow} H$ be $a$ $\mathfrak{B}$-invariant sequence. Then $\pi\left(C_{G}(\mathfrak{B})_{0}\right)=C_{G / K}(\mathfrak{B})_{0}$.

Proof. Write $H:=G / K$. Because of $C_{G}(\mathfrak{B})_{0} \subseteq G_{0}$ we may assume $G=G_{0}$. We distinguish four cases:

(1) $K \in[\mathrm{TD}]$ (in this case, $G$ need not be in $[\mathrm{SIN}]_{\mathfrak{B}}$ );

(2) $K$ and $G$ are connected Lie groups;

(3) $G \in[$ Lie];

(4) the general case.

(1) From the continuity of $\varphi_{\beta}: G \rightarrow G$,

$$
\varphi_{\beta}(g):=g^{\beta} g^{-1}
$$

and

$$
W:=\varphi_{\beta}\left(\left(\pi^{-1}\left(C_{G / K}(\mathfrak{B})\right)\right)_{0}\right) \subseteq K
$$

we infer that $W=E$, i.e., $\left(\pi^{-1}\left(C_{G / K}(\mathfrak{B})\right)\right)_{0} \subseteq C_{G}(\mathfrak{B})_{0}$. An application of $\pi$ yields the result.

(2) In view of the connectedness of $K$ the sequence $K>U_{0} \rightarrow C_{H}(\mathfrak{B})_{0}$, where $U_{0}:=\left(\pi^{-1}\left(C_{H}(\mathfrak{B})_{0}\right)\right)_{0}$, is exact. It suffices to show that $U_{0}=C_{G}(\mathfrak{B})_{0} K$. This, however, follows from the relation (to be proved), $\mathfrak{u}=\mathfrak{c}_{\mathfrak{h}}\left(\mathfrak{B}^{0}\right)+\mathfrak{f}$, involving the Lie algebras $\mathfrak{f}, \mathfrak{u}, \mathfrak{c}_{\mathfrak{h}}\left(\mathfrak{B}^{0}\right)$ of the connected Lie groups $K, U_{0}, C_{H}(\mathfrak{B})_{0}$, where $\mathfrak{B}^{0}$ denotes the canonical image of $\mathfrak{B}$ in Aut $\mathfrak{g}$. Since $G \in[\mathrm{SIN}]_{\mathfrak{B}} \cap[\mathrm{K}]$, it follows that $\mathfrak{B}^{-}$is compact in $\operatorname{Aut}(G)$ and, hence, $\left(\mathfrak{B}^{0}\right)^{-}$is compact in Aut $g$. Hence the restriction image of $\left(\mathfrak{B}^{0}\right)^{-}$in Aut $\mathfrak{U}$ is compact. Therefore there exists a $\mathfrak{B}^{0}$-invariant decomposition $\mathfrak{u}=\mathfrak{l} \oplus \mathfrak{g}$. Clearly, for $s \in \mathfrak{g}, \beta \in \mathfrak{B}, s^{\beta}-s \in \mathfrak{g} \cap \mathfrak{t}=\{0\}$, and therefore $\mathfrak{g} \leqslant \mathfrak{c}_{\mathfrak{u}}\left(\mathfrak{B}^{0}\right)$, i.e. $\mathfrak{c}_{\mathfrak{u}}\left(\mathfrak{B}^{0}\right)+\mathfrak{H}=\mathfrak{u}$ follows.

(3) Apply (1) and (2) to the $\mathfrak{B}$-invariant sequences $K \gg G \rightarrow H, K / K_{0} \rightarrow$ $G / K_{0} \rightarrow H, K_{0} \rightarrow G \rightarrow G / K_{0}$. 
(4) We have $G \in[\mathrm{SIN}]_{\mathcal{B}} \cap[\mathrm{FC}]^{-} \cap[\mathrm{K}]$ so $\mathcal{B}^{-}(\operatorname{Inn} G)$ is compact in Aut $G$ by [5]. According to [4, (2.11)], $G=\lim \operatorname{proj}\left\{G / N_{i}, \varphi_{i k}\right\}$, where each $N_{i}$ is $\mathfrak{B}$-invariant and $G / N_{i}$ is a Lie group. Clearly, $G / N_{i} \in[\mathrm{SIN}]_{W} \cap[\mathrm{K}]$, and, by $(3), \pi_{i}\left(C_{G / N_{i}}(\Re \mathcal{B})_{0}\right)=$ $C_{G / K N_{i}}(\mathscr{B})_{0}$, where $\pi_{i}: G / N_{i} \rightarrow G / K N_{i}$ is the canonical epimorphism induced by $\pi$. Since lim proj is right exact, the final result follows.

(4.2) Theorem. Let $G \in[\mathrm{CF}]^{-}$and $G_{0} \in[\mathrm{SIN}]_{G}$. Then $G$ is either totally disconnected or compact.

Proof. Let $G_{0} \neq E$, but $G$ is noncompact. Since $[\mathrm{P}]^{-} \supset[\mathrm{CF}]^{-}, G_{0}$ is compact [4, (2.13), p. 12]. Let $T$ be a maximal torus in $G_{0}$. Because of (2.4)(2) we have $G=N_{G}(T) G_{0}$; hence $N_{G}(T)$ is noncompact. It follows from $G_{0} \in[\mathrm{SIN}]_{G}$ that $T \in[\mathrm{SIN}]_{N_{G}(T)}$. Since $T$ is compact it follows from $[5,(1.7)$, p. 326] that the canonical image of $N_{G}(T)$ in $\operatorname{Aut}(T)$ is precompact; hence it follows from [4, (2.11), p. 11] that there exists $T_{1} \triangleleft N_{G}(T), T_{1}<T$ with $T_{1} \rightarrow T \rightarrow \overline{\mathbf{T}}^{n}$, where $\overline{\mathbf{T}}^{n}$ is an $n$-dimensional torus, $n \neq 0$. Let $\varphi: N_{G}(T) \rightarrow \operatorname{Aut}\left(\overline{\mathbf{T}}^{n}\right)$ be the canonical homomorphism resulting from the $N_{G}(T)$-invariant sequence above. Since $N_{G}(T)$ is periodic and $T \leqslant \operatorname{Ker} \varphi, \varphi\left(N_{G}(T)\right)$ is a torsion subgroup of $\operatorname{Aut}\left(\overline{\mathbf{T}}^{n}\right) \cong \operatorname{Sl}_{n}(\mathbf{Z})$ and is therefore finite, in view of $[5,(1.13)$, p. 329]. Let $B:=\operatorname{Ker} \varphi$. Then $B$ is closed but not compact, because otherwise $N_{G}(T)$ would have to be compact.

By construction, $\overline{\mathbf{T}}^{n}=C_{\mathbf{T}^{n}}^{-}(B)_{0}$. Now let $\psi: N_{G}(T) \rightarrow$ Aut $\overline{\mathbf{T}}^{n}$ be the canonical homomorphism, $\mathfrak{B}:=\psi(B)$, and let $G:=T, H:=\overline{\mathbf{T}}^{n}$ in (4.1). It follows that $\pi\left(C_{T}(\mathfrak{B})_{0}\right)=\overline{\mathbf{T}}^{n}$, so $C_{T}(\mathfrak{B})_{0} \neq E$. For $x \in\left(C_{T}(\mathfrak{B})_{0} \backslash E\right)$ we therefore have $B \leqslant$ $C_{G}(x)$, i.e., $C_{G}(x)$ cannot be compact, a contradiction.

(4.3) THEOREM. Every Moore group with centralizer condition $[\mathrm{CF}]^{-}$is either compact or a finite extension of a Moore p-group ( $p$ a prime).

The proof will be given in a number of stages. First we need several technical definitions and results concerning groups in $\Omega$. After that we construct a hypothetical counterexample to the conclusion of (4.3).

(4.4) Lemma. For $G \in \Omega$ let $K>G \rightarrow D$ be as in the proof of (2.1) and let $T$ be a closed noncompact subset of $G$. Then there exists a countable subset $A \subseteq T$ with $A K$ $\sigma$-compact and noncompact, and $\langle A\rangle K \sigma$-compact.

Proof. Since $K$ is compact and open, $\{g K \mid g \in G\}$ is an open covering of $G$ (hence of $T$ ) by pairwise disjoint compact sets. Since $T$ is noncompact there are infinitely many $t \in T$ lying in different sets $g K$. Hence there is an infinite countable number of such elements $t$. Let $A$ be such a set. Then $A K$ is open and $\sigma$-compact. Analogously, $A^{r} K$, and therefore also $\langle A\rangle K=\cup_{r \in \mathbf{Z}} A^{r} K$ is $\sigma$-compact.

Definition. Call $G \in \Omega p$-discrete, if there exists a $\sigma$-compact, closed noncompact $p$-subgroup in $G$.

Definition. For $G \in \Omega$ let $M_{p}(G):=\{x \in G \mid x$ is a $p$-element $\}$. (Note that, in view of $(2.1), M_{p}(G)$ is closed.)

Definition. We call $H \leqslant G$ a $\sigma$-subgroup, if the following holds:

(1) $H$ is closed and noncompact; 
(2) $H=\cup_{i \in \mathbf{N}} H_{i}, H_{i}$ open and compact subgroups of $H, H_{i} \leqslant H_{i+1}$.

(4.5) Lemma. Assume there exists $K \gg G \rightarrow A$ with $K$ profinite and open and $A$ an abelian torsion group. Then $G \in \Omega \cap[\mathrm{LF}]^{-} \cap[\mathrm{FD}]^{-}$. If $M_{p}(G)$ is noncompact then there exists a p-o-subgroup $S_{p}$ and $G$ is p-discrete.

Proof. Obviously $G \in \Omega \cap[\mathrm{FD}]^{-}$, because of (2) in (2.2) $G \in[\mathrm{LF}]^{-}$. Since $K$ is compact, $M_{p}(A)$ is noncompact if $M_{p}(G)$ is noncompact. Let $\left\{x_{i} \mid i \in \mathbf{N}\right\}$ be a countable subset of a system of representatives of $G / K$ contained (see (4.4)) in $M_{p}(G)$; let

$$
H:=\left\langle K,\left\{x_{i}\right\}\right\rangle, \quad H_{k}:=\left\langle K,\left\{x_{1}, \ldots, x_{k}\right\}\right\rangle .
$$

Each $H_{k}$ is open and $H=\cup_{k \in \mathrm{N}} H_{k}$. By passing, if necessary, to a subsequence of $\left\{x_{i}\right\}$, one may assume $H_{k}<H_{k+1}$. Thus $H$ is an open $\sigma$-subgroup. Let $S_{p}\left(H_{1}\right)$ be a $p$-Sylow-subgroup of $H_{1}$. Since $H_{k+1}$ is profinite for every $k, S_{p}\left(H_{1}\right)$ can be extended to a $p$-Sylow-subgroup $S_{p}\left(H_{2}\right)$. One obtains the ascending sequence $\left\{S_{p}\left(H_{k}\right) \mid k \in \mathbf{N}\right\}$ and $S_{p}:=\cup_{k \in \mathbf{N}} S_{p}\left(H_{k}\right)$. By construction $H_{k+1} / H_{k}$ is a nontrivial finite $p$-group. Thus there exists a $p$-element $t_{k} \in H_{k+1} \backslash H_{k}$. Hence there exists $y_{k} t_{k} y_{k}^{-1} \in$ $\left(S_{p}\left(H_{k+1}\right) \backslash S_{p}\left(H_{k}\right)\right)$. Obviously, $t_{k} \in S_{p}$ is not totally bounded, so $S_{p}$ is not compact. One has $H_{k} \cap S_{p}=S_{p}\left(H_{k}\right)$. Since $H_{k}$ is compact, $S_{p} \cap H$ is closed. Let $\left\{x_{\nu} \mid \nu \in \mathbf{N}\right\}$ be a net in $S_{p}$ converging to $x$. Then $x \in H$ and therefore $x \in H_{k}$ for some $k$. Since $H_{k}$ is open it follows that $x_{\nu} \in H_{k}$ for $\nu \geqslant \nu(k)$. Therefore, $x_{\nu} \in H_{k} \cap S_{p}=S_{p}\left(H_{k}\right)$. Since the latter group is compact, it follows that $x \in S_{p}\left(H_{k}\right) \subseteq S_{p}$. Thus $S_{p}$ is closed.

(4.6) Lemma. Assume $G \in \Omega \cap[\mathrm{LF}]^{-} \cap[\mathrm{FD}]^{-}$. Then the following holds.

(1) $L \unlhd G$ and $L$ compact $\Rightarrow(G$ is $p$-discrete $\Leftrightarrow G / L$ is p-discrete $)$.

(2) $G=H L, L \leqslant G, L$ compact $\Rightarrow(G$ is p-discrete $\Leftrightarrow H$ is p-discrete $)$.

(3) $L \gg G \rightarrow T$ with $T$ compact $\Rightarrow(G$ is p-discrete $\Leftrightarrow L$ is p-discrete $)$.

Proof. (1) We have the following two sequences: $L \gg G \rightarrow G / L$ with $L$ compact; $K \gg G \rightarrow G / K$ with $K$ compact and open, $G / K$ a discrete abelian torsion group.

$(\Rightarrow)$ Let $S_{p}$ be a $p$ - $\sigma$-subgroup of $G$. From the first sequence one sees that $S_{p} L / L$ is a $p$ - $\sigma$-subgroup of $G / L$.

$(\Leftarrow)$ Let $S_{p}^{*}:=S / L$ be a $p$ - $\sigma$-subgroup of $G / L$. We first show that $S /(S \cap K) L$ $\cong(S / L) /(((S \cap K) L) / L)$ is an infinite discrete (locally finite) $p$-group. Now $((S \cap K) L) / L$ is a compact open subgroup of $S / L=S_{p}^{*}$, so $S /(S \cap K) L$ is a discrete $p$-group. If it were finite then $S_{p}^{*}$ would have to be compact, a contradiction. Thus it immediately follows from the sequence $(S \cap K) L>S \rightarrow S /(S \cap K) L$ that $M_{p}(S)$ is noncompact. An application of (4.5) to the sequence $S \cap K \gg$ $S \rightarrow S /(S \cap K)$ yields a $p$ - $\sigma$-subgroup $S_{p}$ of $S$, hence one of $G$.

(2) First note that $G / L K=H L K / L K \cong H / H \cap(L K)$. Because of (1) one also has

$H p$-discrete $\Leftrightarrow H / H \cap(L K) p$-discrete $\Leftrightarrow G / L K p$-discrete $\Leftrightarrow G p$-discrete.

(3) ( $\Rightarrow$ ) In the sequence $L K \gg G \rightarrow T_{1}, T_{1}$ is compact abelian and discrete, hence finite. Therefore, and because of (1), $G / K$ is $p$-discrete and abelian in the sequence 
$L K / K \supset G / K \rightarrow T_{1}$, hence contains an infinite abelian $p$-subgroup $H / K$. Hence, because $T_{1}$ is finite, there exists an infinite abelian $p$-subgroup in $L K / K$. Therefore, $L K / K \cong L / L \cap K$ is $p$-discrete. From (1) it follows that $L$ is $p$-discrete.

(4.7) Lemma. Assume $G \in \Omega, G=B H, B=B^{-} \leqslant G, H \leqslant G, H$ compact, $K=K^{-}$ $\leqslant H, B \leqslant N_{G}(K), B=\cup_{i \in \mathrm{N}} B_{i}, B_{i+1} \geqslant B_{i}$, each $B_{i}$ compact and open in $B$, and $\Pi(B) \cap \Pi(K)=\Phi$. If there exists $h \in H$, such that $(h K)^{B}=h K$, then there exists $x \in h K$ with $x^{B}=x$, i.e., $B \leqslant C_{G}(x)$.

Proof. Each $B_{i} H$ is compact, hence profinite. Because of $(h K)^{B_{t}}=h K, \Pi\left(B_{i}\right) \subseteq$ $\Pi(B)$ and $\Pi(B) \cap \Pi(K)=\Phi$ it follows from [3, Lemma 1.3] that there exists a sequence $\left\{x_{i}\right\} \subseteq h K$ with $x_{i}^{B_{i}}=x_{i}$ for all $i \in \mathbf{N}$. Let $x$ be a clusterpoint of the sequence $x_{i}$ in the compact set $h K$. W.l.o.g. assume $\lim x_{i}=x$. For $b \in B$ we have $b \in B_{j}$ for some $j$. Let $i \geqslant j$, so $B_{i} \supseteq B_{j}$. Then $x_{i}^{B_{j}}=x_{i}$, therefore $x^{B_{i}}=x$, hence $x^{B}=x$.

(REMARK. The condition stated in 1.3 of [3] ought to read $(|A|,|H|)=1$.)

Definition. $G \in\left[\mathrm{F}_{p}\right]: \Rightarrow G$ is profinite or is a finite extension of a $p$-group $P$ in $\Omega: P>G \rightarrow G / P$.

(4.8) Lemma. $N \gg G \rightarrow F$ with $F$ finite $\Rightarrow\left(G \in\left[\mathrm{F}_{p}\right] \Leftrightarrow N \in\left[\mathrm{F}_{p}\right]\right)$.

Proof. If $G$ or $N$ is profinite, we are done.

$(\Rightarrow)$ From $P>G \rightarrow G / P$ we deduce the sequence $N \cap P>N \rightarrow N P / P$. Since $\Omega$ is stable with respect to the passage to closed subgroups, it follows that $N \cap P \in \Omega$.

$(\Leftarrow)$ Assume we have $P_{1} \longrightarrow N \rightarrow N / P_{1}$ with $P_{1}$ a $p$-group in $\Omega$ and $N / P_{1}$ finite. $\left[G: P_{1}\right]$ is finite and, therefore, the normal subgroup $P:=\cap_{g \in G} P_{1}^{g}$ is of finite index in $G$ because it is a finite intersection of subgroups of finite index. From $P \leqslant P_{1} \leqslant N$ we infer $P \in \Omega$.

Proof OF (4.3). Because of (4.2) it suffices to assume $G \in[T D]$. For the proof by contradiction we now assume

$$
G \in([\mathrm{M}] \cap[\mathrm{TD}]) \backslash \bigcup_{p}\left(\left[\mathrm{~F}_{p}\right]\right) .
$$

Claim 1. $G$ may be assumed to be of the form $K \supset G \rightarrow A$, where $K$ is a profinite $[\mathrm{SIN}]_{G}$-group, and $A$ is a discrete abelian torsion group.

Proof. According to [11, Theorems 2, 3, p. 402] one has $G=\lim \operatorname{proj} G_{\alpha}$, where, in the sequence $K_{\alpha} \longrightarrow G \rightarrow G_{\alpha}, K_{\alpha}$ is compact. Furthermore, $M_{\alpha} \longrightarrow G_{\alpha} \stackrel{\pi_{\alpha}}{\rightarrow} F_{\alpha}$, where $M_{\alpha}$ is a discrete [Z]-group, $F_{\alpha}$ is finite and $G_{\alpha}$ is a discrete [M]-group. (Compare also [18].) Put $A_{\alpha}:=\pi_{\alpha}^{-1}\left(Z\left(M_{\alpha}\right)\right.$ ) and let $K_{\alpha} \rightarrow A_{\alpha} \rightarrow Z\left(M_{\alpha}\right)$ be the corresponding sequence. Since $G / A_{\alpha}$ is finite one may replace $G$ by $A_{\alpha}$ in view of (4.8).

Claim 2. $p \in \Pi(G) \Rightarrow M_{p}(G)$ is compact.

Proof. Assume $M_{p}(G)$ is noncompact. If $G$ contains no $q$-element, $q \neq p$, then $G$ is a $p$-group, a contradiction. Let $x_{0}$ be a $q$-element. Replacing $K$, if necessary (see Claim 1), by $\left\langle x_{0}, K\right\rangle=\left\langle x_{0}\right\rangle^{-} K$, one may assume $x_{0} \in K$. Then there exists a nontrivial $q$-Sylow subgroup $Q$ of $K$. It follows from (2.4)(1) that $G=N_{G}(Q) K$. 
Since $M_{p}(G)$ is noncompact it follows that $G$ is $p$-discrete $((4.5))$; from this it follows that $M_{p}\left(N_{G}(Q)\right)$ is noncompact so $N_{G}(Q)$ is $p$-discrete $(4.6)(2)$. Hence there exists ((4.5)) a $p$ - $\sigma$-group $P=P^{-} \leqslant N_{G}(Q)$. Since $K \in[\mathrm{SIN}]_{G}$ it follows from $G=N_{G}(Q) K$ that $Q \in[\mathrm{SIN}]_{P}$. Since $Q$ is profinite, there exists $Q_{1}<Q, Q_{1}^{P}=Q_{1}, 1<\left|Q / Q_{1}\right|<$ $\boldsymbol{\aleph}_{0}$. Thus there is a continuous homomorphism $\varphi: P \rightarrow \operatorname{Aut}\left(Q / Q_{1}\right)$. For $P_{1}:=\operatorname{Ker} \varphi$ we then have $\left(y Q_{1}\right)^{P_{1}}=y Q_{1}$ for all $y \in Q$. Let $y \in Q \backslash Q_{1} . P_{1}$ is also a $p$ - $\sigma$-group. On putting $B:=P_{1}, H:=Q, K:=Q_{1}, h:=y$ in (4.7) one sees there exists $x \in y Q_{1}$ with $P_{1} \leqslant C_{G}(x)$. Since $P / P_{1}$ is finite, $P_{1}$ is not compact. Since $P_{1}$ is closed, a contradiction results.

Claim 3. $\Pi(G / K)=\boldsymbol{\aleph}_{0}$.

Proof. Assume $\Pi(G / K)=\left\{p_{1}, \ldots, p_{n}\right\}$. We show that $M_{p_{i}}(G) K / K=M_{p_{i}}(G / K)$. Clearly $\subseteq$ holds. Let $x \in G, \pi(x) \in M_{p_{1}}(G / K)$. Now (by Claim 1) $\langle x, K\rangle=\langle x\rangle K$ is profinite and $\pi\langle x, K\rangle=\langle\pi(x)\rangle$ is finite. Hence there exists $y \in M_{p_{1}}(\langle x, K\rangle)$ with $\pi(y)=\pi(x)$. On account of $M_{p_{i}}(\langle x, K\rangle) \subseteq M_{p_{i}}(G)$, one has $M_{p_{i}}(G / K) \subseteq$ $M_{p_{i}}(G) K / K$. Hence $M_{p_{t}}(G / K)$ is finite (by Claim 2) so $G / K$ is finite and therefore $G$ compact, a contradiction.

(4.3) (END of Proof). $G \in[\mathrm{CF}]^{-} \Rightarrow G \in[\mathrm{AF}]^{-}$. Because of $(2.5)$, for $D:=G / K$, it follows that $|\Pi(G / K)|<\boldsymbol{\aleph}_{0}$, a contradiction to Claim 3.

\section{Examples and remarks.}

(5.1) EXAMPLE (Nilpotent group, of class 2, in $[\mathrm{AF}]^{-} \backslash[\mathrm{CF}]^{-}$). Let $p$ be an odd prime, $W:=C_{p}^{\mathbf{N}}$, with its natural compact topology. $V:=C_{p}^{\mathbf{N}}$ endowed with the discrete topology. Let $G:=V \times \Lambda^{2}(W)$ with the operation

$$
(v, \sigma)\left(v^{\prime}, \sigma^{\prime}\right):=\left(v+v^{\prime}, \sigma+\sigma^{\prime}+2^{-1} v \wedge v^{\prime}\right) .
$$

$G$ is a nilpotent group of class 2 . Topologize $G$ by the sequence $\Lambda^{2}(W)>G \rightarrow V$, in which $\Lambda^{2}(W)$ is clearly compact. From the commutator relation $\left[(v, \sigma),\left(v^{\prime}, \sigma^{\prime}\right)\right]$ $=\left(0, v \wedge v^{\prime}\right)$, one sees that $\Lambda^{2}(W)=Z(G)$. Since $G$ is not compact, the elements of $Z(G)$ violate the condition $[C F]^{-}$. It is easy to see that, for $v \in V \backslash\{0\}$,

$$
C_{G}(v, \sigma)=\left\{(r v, \tau) \mid r \in \operatorname{GF}(p), \tau \in \Lambda^{2}(W)\right\},
$$

so $C_{G}(v, \sigma)$ is an abelian group. It follows that $C_{G}(v, \sigma)$ is compact for $v \neq 0$. Since every maximal abelian subgroup of $G$ contains $Z(G)$, every such subgroup is of the form $C_{G}(v, \sigma)$ for a $v \neq 0$. Thus $G \in[\mathrm{AF}]^{-}$.

(5.2) EXAMPLE (A noncompact Moore group with centralizer condition). Let $F:=\left\langle x_{1}, x_{2}, \ldots\right\rangle$ be the free group in countably many variables $\left\{x_{i}\right\}$, and let $p$ be a fixed prime. The system $\Phi:=\left\{N \leqslant F \mid x_{i} \in N\right.$ for almost all $i$ and $[F: N]=p^{k}$ for some $k \in \mathbf{N}\}$ of normal subgroups constitutes a fundamental system of $e$-neighborhoods in $F$; in the resulting topology the $x_{i}$ converge toward $e$. Let $\hat{F}_{p}$ be the completion of $F$ with respect to this topology. $\hat{F}_{p}$ is the restricted free pro-p-group in countably many variables (see [15]). For its Frattini subgroup $\hat{F}_{p}^{*}$ one has $\hat{F}_{p}^{*}=\hat{F}_{p}^{p} \hat{F}_{p}^{\prime}$. Let $G:=F \cdot \hat{F}_{p}^{*}$. This gives rise to the algebraic sequence $\hat{F}_{p}^{*} \rightarrow G \rightarrow\left(C_{p}^{\mathbf{N}}\right)^{*}$, by means of which $G$ is topologized as a locally compact, noncompact group, with $\hat{F}_{p}^{*}$ as a compact open subgroup. We show that $G \in[\mathrm{M}] \cap[\mathrm{LF}]^{-}$. (Instead of our original proof of this assertion we offer a short proof due to G. Schlichting.) Let $K \in[\mathrm{K}]$ and 
let $K \supseteq G \supseteq L \supseteq\left(K^{\prime}\right)^{-}$be a sequence of subgroups of $K$ such that $L$ is a closed normal subgroup of $G$. One may define a new topology on $G$ by the sequence $L>G \rightarrow G / L$, where $L$ is open and compact.

Since $G>K$ is continuous, we have $G \in[\mathrm{MAP}]$. Since, obviously, $G \in[\mathrm{FD}]^{-}$, it follows from known results on compactness conditions [13, p. 701] that $G \in[\mathrm{M}]$.

Call $z$ a primitive element for $x$, if $x=x^{p^{k}}$, but if no $t$ with $x=t^{p^{k+1}}$ can be found. Note also that each $x \in \hat{F}_{p}$ has a unique representation $x=\left(\prod_{i=1}^{\infty} x_{i}^{\alpha_{i}}\right) \eta$, with $\alpha_{i} \in \mathbf{Z}_{p}$ ( $p$-adic integers) and $\eta \in \hat{F}_{p}^{\prime}$ (this is seen by passing from $\hat{F}_{p}$ to $\hat{F}_{p} / \hat{F}_{p}^{\prime}$ ). We first note that $\mathbf{Z}_{p}$ is a compact integral domain containing $\mathbf{Z}$ as a dense subring. Accordingly one says that $p \mid \alpha$, if there is a $\beta \in \mathbf{Z}_{p}$ with $\alpha=p \beta$. By passing to $\hat{F}_{p} / \hat{F}_{p}^{\prime}$ one observes from the construction of $G$, that $x \in G$ if and only if $x=\left(\prod_{i=1}^{\infty} x_{i}^{\alpha_{t}}\right) \eta$, $\eta \in \hat{F}_{p}^{\prime}$, and almost all $\alpha_{i}$ are divisible by $p$. According to this we have $x \in \hat{F}_{p}^{*}$ if and only if every $\alpha_{i}$ is divisible by $p$.

For the proof of the compactness of $C_{G}(x)$, let

$$
z=\left(\prod_{i=1}^{\infty} x_{i}^{\beta_{i}}\right) \eta_{1}, \quad \eta_{1} \in \hat{F}_{p}^{\prime},
$$

be a primitive element for $x$. According to [3, Lemma 4.1, p. 526] we have $C_{\hat{F}_{p}}(x)=\langle z\rangle^{-}$, where the closure is taken in $\hat{F}_{p}$. Hence $C_{G}(x)=\langle z\rangle^{-} \cap G$. If almost all $\beta_{i}$ are divisible by $p$ then, for $z \in G$ and $n$ sufficiently large, it follows that

$$
\langle z\rangle^{-} \cap G \leqslant\left\langle x_{1}, \ldots, x_{n}\right\rangle^{-} \hat{F}_{p}^{*}
$$

hence $C_{G}(x)$ is compact. If, however, infinitely many $\beta_{i}$ are not divisible by $p$, then $z \notin G$ holds. From this it follows that $z^{r} \notin G$ for $0<r<p$, because

$$
z^{r}=\left(\prod_{i=1}^{\infty} x_{i}^{r \beta_{i}}\right) \eta(r), \quad \text { where } \eta(r) \in \hat{F}_{p}^{\prime} \text { and } p\left|\beta_{i} \Leftrightarrow p\right| r \beta_{i}
$$

holds. In particular, this implies $z^{r} \hat{F}_{p}^{*} \cap G=\varnothing$ if $0<r<p$. We have, however, the coset decomposition

$$
\langle z\rangle^{-} \hat{F}_{p}^{*}=\bigcup_{r=0}^{p-1} z^{r} \hat{F}_{p}^{*}
$$

of $C_{\hat{F}_{p}}(x) \hat{F}_{p}^{*}$. It follows that

$$
C_{G}(x)=\langle z\rangle^{-} \cap G \leqslant\left(\langle z\rangle^{-} \hat{F}_{p}^{*}\right) \cap G=\bigcup_{r=0}^{p-1} z^{r} \hat{F}_{p}^{*} \cap G=\hat{F}_{p}^{*},
$$

and $C_{G}(x)$ is compact.

REMARK. One can construct examples of groups $G \in[\mathrm{AF}]^{-} \cap[\mathrm{M}]$ with $G_{0} \neq E$. Thus (4.2) cannot be generalized to this class. It is our conjecture that (4.2) holds for $G \in[\mathrm{AF}]^{-} \cap[\mathrm{SIN}] \cap[\mathrm{LF}]^{-}$. Finally, another open problem is suggested by the well-known examples of groups due to Olshanskij [12]-infinite groups all of whose proper subgroups are finite. Such groups may be viewed as examples of noncompact [SIN]-groups all of whose proper closed subgroups are compact. Are there nondiscrete [SIN]-groups with this property which are not compact? 


\section{REFERENCES}

1. S. I. Adian, The Burnside problem and identities in groups (translated by J. Lennox and J. Wiegold), Springer-Verlag, Berlin and New York, 1979.

2. L. Fuchs, Infinite abelian groups, Vol. 2, Academic Press, New York, 1970.

3. D. Gildenhuys, W. Herfort and L. Ribes, Profinite Frobenius groups, Arch. Math. (Basel) 33 (1979), $518-528$

4. S. Grosser and M. Moskowitz, Compactness conditions in topological groups, J. Reine Angew. Math. 246 (1971), 1-40.

5. S. Grosser, O. Loos and M. Moskowitz, Über Automorphismengruppen lokal-kompakter Gruppen und Derivationen von Lie-Gruppen, Math. Z. 114 (1970), 321-339.

6. W. Herfort, Compact torsion groups and finite exponent, Arch. Math. (Basel) 33 (1979), 404-410.

7. G. Hochschild, The structure of Lie groups, Holden-Day, San Francisco, Calif., 1969.

8. O. H. Kegel and B. A. F. Wehrfritz, Locally finite groups, North-Holland, Amsterdam and London; American Elsevier, New York, 1973.

9. O. Loos, Symmetric spaces. II, Benjamin, New York, Amsterdam, 1969.

10. J. R. McMullen, Compact torsion groups, Proc. Second Internat. Conf. Theory of Groups, Lecture Notes in Math., Springer, Berlin and New York, 1973.

11. C. C. Moore, Groups with finite-dimensional irreducible representations, Trans. Amer. Math. Soc. 166 (1972), 401-410.

12. A. Yu. Olshanskij, An infinite group with its subgroups of prime order, Izv. Akad. Nauk SSSR Ser. Mat. 44 (1980), 309-321.

13. T. W. Palmer, Classes of nonabelian noncompact locally compact groups, Rocky Mountain J. Math. 8 (1978), 683-741.

14. V. P. Platonov, Periodic and compact subgroups of topological groups, Sibirsk. Mat. Ž. 7 (1966), 854-877. (Russian)

15. L. Ribes, Introduction to profinite groups and Galois cohomology, Queen's Papers in Pure and Appl. Math. vol. 24, Queen's Univ., Kingston, Ont., 1970.

16. D. J. S. Robinson, Finiteness conditions and generalized soluble groups, part 1, Springer, Berlin and New York, 1972.

17. J.-P. Serre, Cohomologie Galoisienne, Lecture Notes in Math., vol. 5, Springer, Berlin, 1965.

18. E. Thoma, Über unitäre Darstellungen abzählbarer diskreter Gruppen, Math. Ann. 153 (1964), $111-132$.

InStitut für Mathematik, Universität Wien, A-1090 Wien, AUSTRia

InStitut 115, TechNische UNiversität Wien, A-1040 WiEN, AUSTRIA 